\title{
Prática de atividade física em adolescentes brasileiros
}

\author{
Physical activity practice among Brazilian adolescents
}

Pedro Curi Hallal ${ }^{1}$

Alan Goularte Knuth ${ }^{2}$

Danielle Keylla Alencar Cruz ${ }^{3}$

$M$ aria I sabel $M$ endes ${ }^{4}$

Deborah Carvalho $\mathrm{M}$ alta ${ }^{3}$

\footnotetext{
${ }^{1}$ Escola Superior de Educação Física, UniversidadeFederal de Pelotas. Rua M arechal Deodoro 1.160, 30 piso. 96020-220 Pelotas RS. prchallal@gmail.com 2 Programa de PósGraduação em Epidemiologia UniversidadeFederal de Pelotas.

${ }^{3}$ Coordenação Geral de Doenças e Agravos Não Transmissíveis, M inistério da Saúde.

${ }^{4}$ Instituto Brasileiro de Geografia e Estatística.
}

Abstract The aim of this study is to describe physical activity practice among Brazilian adolescents by using data from the National Adolescent School-based Health Survey (PeN SE) collected in all state capitals and the Federal District in 2009. Thesampleincluded students of theninth year $(n=60,973)$. It was investigated the physical activity practice seven days prior to the interview, including transportation mode, physical activity practiceinside and outside theschool and participation in Physical Education classes. Adolescentswereclassified as active if performing $\geq 300$ $\mathrm{min} / \mathrm{wk}$ of physical activity. The proportion of activeadolescentswas $43.1 \%$; thisfigurewashigher among boys ( $56.2 \%)$ as compared to girls $(31.3 \%)$. Only half of the adolescents (49.2\%) reported that had two or more Physical Education classes in the week prior to the interview. Also, $79.2 \%$ reported watching TV for two or more hours per day. Our data indicate a low proportion of active adolescents and of regular Physical Education classes, and a high prevalence of sedentary behavior. These data might be used as a baseline for the monitoring of physical activity among adolescents in Brazil, but al ready suggests the need of interventions for the promotion of physical activity among Brazilian adolescents. Key words M otor activity, Population surveillance, Adolescent, Sedentary lifestyle
Resumo 0 objetivo desteestudo édescrever a prática de atividade física em adolescentes, utilizando dados da Pesquisa Nacional de Saúde do Escolar (PeN SE), coletadosem todas as capitais do Brasil e no Distrito Federal em 2009. A amostra incluiu escolares do 90 ano do ensino fundamental $(n=60.973)$. Foram investigadas a prática de atividadefísica nosúltimos sete dias, incluindo modo de deslocamento para a escola, prática de atividade física dentro e fora da escola eparticipação nas aulas de educação física. Consideraram-se ativos os jovens que acumularam $\geq 300 \mathrm{~min} / \mathrm{sem}$ de atividade física. A proporção de ativos foi de 43,1\%, sendo maior nos meninos $(56,2 \%)$ em comparação às meninas (31,3\%). M etade dos adolescentes $(49,2 \%)$ relatou ter tido duas ou mais aulas de educação física na semana anterior à entrevista; $79,2 \%$ relataram assistir a duas horas diárias de televisão ou mais. Os dados do PeNSE indicam baixa prevalência de jovens ativos e com duas ou mais aulas de educação física por semana, além de elevada prevalência de comportamento sedentário. Tais dados podem ser utilizados como linha de base para o monitoramento de atividadefísica em escolares brasileiros, mas desdejá sugerem a necessidade de intervenções para a promoção de atividade física em adolescentes brasileiros. Palavras-chave Atividademotora, M onitoramento populacional, Adolescente, Comportamento sedentário 
Introdução

A prática de atividade física na adolescência está relacionada com benefícios tanto a curto quanto a longo prazo para a saúde. ${ }^{1}$ Além dos efeitos diretos sobre a saúde, a atividade física na adolescência está relacionada com maior probabilidade de prática de atividade física na idade adulta, ${ }^{2}$ gerando um efeito indireto sobre a saúde futura. A pesar do acúmulo de conhecimento científico acerca dos benefícios da atividade física na adolescência para a saúde, diversos levantamentos nacionais apontam para baixos percentuais de jovens ativos ${ }^{3-5}$. Ainda mais preocupantes são as evidências, embora exclusivas de países desenvolvidos, de que a prática de atividade física na adolescência está diminuindo com o passar do tempo, assim como a participação em aulas de educação física e a aptidão física. ${ }^{6}$

0 monitoramento da atividade física em jovens é uma realidade em alguns países desenvolvidos. Nos Estados Unidos, por exemplo, o Youth Risk Behavior Surveillance System (YRBSS) monitora a atividadefísica de adolescentes desde 19917,. No Brasil, o monitoramento da prática de atividade física de adultos foi iniciado com 0 Sistema de Vigilância de Fatores de Risco e Proteção para D oenças Crônicas por Inquérito Tele fônico (Vigitel) em $2006^{\circ}$, e dados anuais têm sido coletados desde então.

A Pesquisa Nacional deSaúde do Escolar (PeNSE) vem preencher algumas lacunas na saúde pública brasileira, especificamente com relação à prática de atividadefísica. Em primeiro lugar, seus dados transversais coletados em 2009 representam o primeiro estudo de base nacional sobre a prática deatividadefísica em adolescentes ${ }^{10}$. Além disso, os dados servirão como linha de base para o monitoramento da prática de atividade física em adolescentes brasileiros. 0 objetivo do pre senteartigo é descrever a prática deatividadefísica, o tempo despendido assistindo televisão e a participação em aulas de educação física de escolares do 9 o ano do ensino fundamental.

\section{Métodos}

Os dados deste estudo são provenientes da Pesquisa Nacional de Saúde do Escolar (PeNSE), uma parceria entre o M inistério da Saúde e o Instituto Brasileiro de Geografia e Estatística (IBGE). 0 objetivo geral da PeN SE foi investigar fatores de risco e proteção à saúde de adolescentes brasileiros. A população-alvo incluiu alunos do 9o ano do ensino fundamental de escolas públicas e privadas de todas as capitais brasileiras e do Distrito Federal.

Como o cadastro refere-se ao conjunto das escolas brasileiras disponível no ano de2007, não foi possível realizar uma seleção direta de uma amostra de escolares. Assim, procedeu-se a um plano com amostragem de conglomerados em dois estágios, em que as unidades amostrais primárias foram as escolas e as secundárias foram as turmas das escolas selecionadas. A amostra de escolares foi formada, portanto, por todos os escolares das turmas sel ecionadas na amostra de escolas. As escolas foram estratificadas, inicialmente, levando-se em conta sua localização geográfica e dependência administrativa. Detalhes adicionais sobre o processo amostral da PeNSE podem ser obtidos em outras publicações ${ }^{11}$.

0 instrumento utilizado para investigar a prática de atividade física foi concebido com baseem instrumentos previamente validados eusados em pesquisas nacionais, incluindo os questionários do Global School-Based Student H ealth Survey ${ }^{12}$, do Youth Risk Behavior Surveillance System ${ }^{7}$, do Estudo sobre a Condição de Saúde e Nutrição dos Escolares da Cidade do Rio de Janeiro, ${ }^{4}$ do Inquérito de Tabagismo em Escolares ${ }^{13}$ edos questionários usados nos acompanhamentos das coortes de nascimento de Pelotas, RS 3 .

A prática deatividadefísica nos sete dias anteriores à entrevista foi investigada. Os adolescentes foram inicialmente questionados sobre 0 modo de deslocamento para a escola, incluindo a duração de tais deslocamentos, por meio da seguinte pergunta: "Nosúltimos sete dias, em quantos dias vocêfoi a péou debicicleta para a escola?" Pergunta idêntica foi feita em relação ao retorno da escola. Além disso, questionou-se o tempo de tais deslocamentos: "Q uando você vai a pé ou de bicicleta para a escola, quanto tempo vocêgasta?" Posteriormente, os jovens foram questionados sobre o número de aulas de educação física que tiveram na semana anterior à entrevista. "Nos últimos setedias, quantas vezes vocêteveaulas de educação física na escola?" Logo após, os jovens responderam sobre a prática de outras atividades físicas, tanto fora quanto dentro da escola. "Nos últimos sete dias, sem contar as aulas de educação física da escola, em quantos dias você praticou al guma atividade física, como esportes, dança, ginástica, musculação, lutas ou outra atividade com a orientação de professor ou instrutor"?"Normalmente, quanto tempo por dia duram essas atividades que você faz com professor ou instrutor?" "Nos últimos sete dias, no seu 
tempo livre, em quantos dias você praticou atividade física ou esporte sem professor ou instrutor?" "Normalmente, quanto tempo por dia duram essas atividades que você faz sem professor ou instrutor"? Por fim, os jovens foram questionados sobre o número de horas diárias despendidos assistindo televisão: "Num dia de semana comum, quantas horas por dia vocêassisteà TV?"

Definiu-se como ativo o adolescente que somou trezentos minutos por semana ou mais de prática de atividade física, somando-se todos os domínios: aulas de educação física, deslocamento para a escola (ida e volta), atividade física fora edentro da escola (atividades extraescolares, tempo livre, outras) ${ }^{14}$. Também foram estimados: (1) o percentual de jovens inativos (não pratica atividade física); (2) que assistem a duas ou mais horas de televisão por dia; (3) que tiveram duas ou mais aulas de educação física na semana anterior à entrevista.

A análise de dados incluiu o cálculo da prevalência de jovens ativos por capital, a proporção de jovens em cada grupo de atividade física (inativos, insuficientemente ativos e ativos) por região e capital do país, e a prevalência dos três desfechos principais (atividadefísica, tempo despendido assistindo televisão por dia e participação em aulas de educação física na semana anterior à entrevista) conforme sexo do adolescente, escolaridade materna (relatada pelo adolescente) e dependência administrativa escolar (pública ou privada). Para as associações envolvendo dependência administrativa escolar e os desfechos, realizamos análise ajustada para escolaridade materna.

A PeN Se foi registrada e aprovada na Comissão Nacional de Ética em Pesquisa (Conep) com o no 11.537. Várias medidas foram tomadas para proteger 0 adolescente e

deixá-lo confortável para responder à pesquisa. A participação foi voluntária; o escolar tinhaa possibilidade de deixar de responder a qualquer pergunta ou a todo o questionário. Todas as informações individuais do escolar foram mantidasem sigilo. A escola também não foi identificada. A realização da pesquisa foi precedida do contato com as Secretarias Estaduais e M unicipais de Saúde e Educação e com a direção das escolas selecionadas em cada município.

\section{Resultados}

Foram entrevistados 60.973 jovens brasileiros nas capitais e no Distrito Federal. Quase $90 \%$ dos jovens frequentando $09^{\circ}$ ano tinham entre $13 \mathrm{e}$ 15 anos de idade. Em relação às principais variáveis em estudo, o tempo assistindo à TV teve 1.164 valores ignorados. $\mathrm{N}$ a variável sobre aulas semanais de educação física, houve 1.034 valores ignorados. Quase 17\% dos alunos não souberam informar a escolaridade materna.

A proporção de jovens ativos foi de $43,1 \%$, sendo maior nos meninos (56,2\%) em comparação às meninas (31,3\%). A penas metade dos adolescentes $(49,2 \%)$ relatou ter tido duas ou mais aulas de educação física na semana anterior à entrevista, e 79,2\% relataram assistir a duas horas diárias de televisão ou mais.

0 percentual de jovens praticando trezentos minutos por semana ou mais de atividade física variou de 34,2\% em São Luís (M A) a 51,5\% em Florianópolis (SC). A penas duas capitais (Florianópolis e Curitiba-PR) tiveram mais da metade dos jovens atingindo as recomendações atuais referentes à prática de atividade física. Em nove capitais, o percentual de jovens ativos foi inferior a 40\% (Gráfico 1).

O Gráfico 2 apresenta a classificação dos adolescentes em níveis deatividadefísica conformea região do país. A maior parte dosjovens do Centro-Oeste, do Sudeste, do Nordeste e do Norte são insuficientemente ativos. Na região Sul, na maioria os jovens foram considerados ativos. 0 percentual de jovens inativos variou de 1,8\% na região Sul a 7,3\% na região N ordeste.

Jovens do sexo feminino foram classificadas como inativas mais frequentemente do que seus pares do sexo masculino. Houve uma associação inversa entre proporção de jovens inativos e escolaridade materna. As diferenças nos níveis de atividade física entre meninos e meninas foram maiores do que aquelas observadas em relação à escolaridade materna (Tabela 1).

Assistir televisão por duas horas ou mais por dia é um comportamento exibido por uma proporção similar de meninos e meninas (Tabela 2). M esmo nos quatro grupos de escolaridade materna, em quea diferença foi estatisticamentesignificativa, a variação na proporção de jovens que assistem à TV duas horas ou mais por dia foi muito pequena ( $77,1 \%$ versus $81,6 \%$ ). Da mesma forma, diferença por tipo de escola foi pequena, $\mathrm{e}$ desapareceu quando foi feito o ajuste para escolaridade materna (dados não mostrados). 
$\%$ jovens ativos

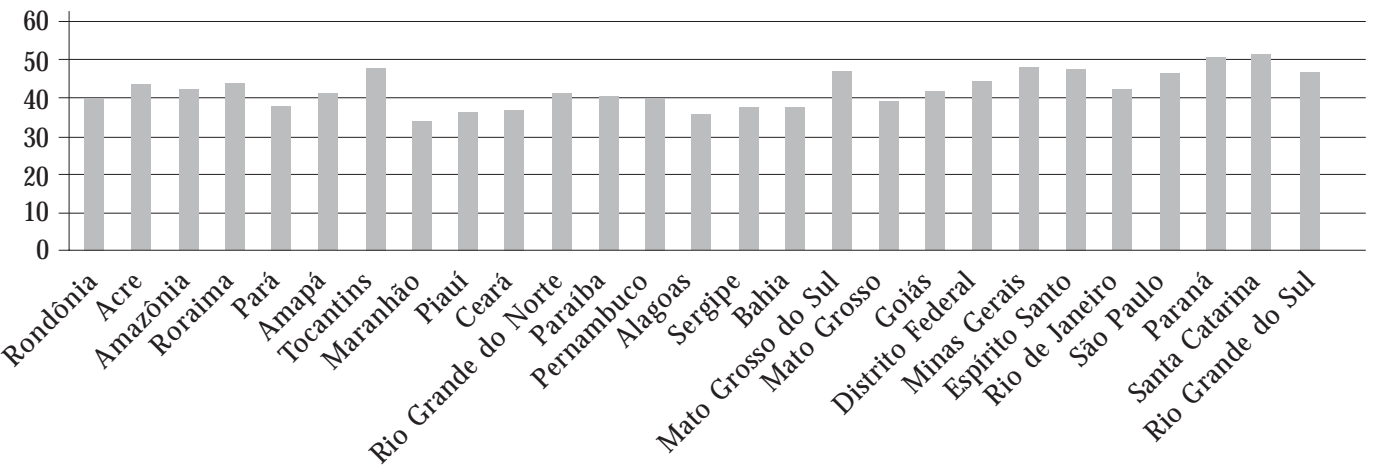

Gráfico 1. Proporção de jovens ativos (> 300 minutos por semana) nas capitais de estado brasileiras e no Distrito Federal. Pesquisa Nacional de Saúde do Escolar, IBGE.

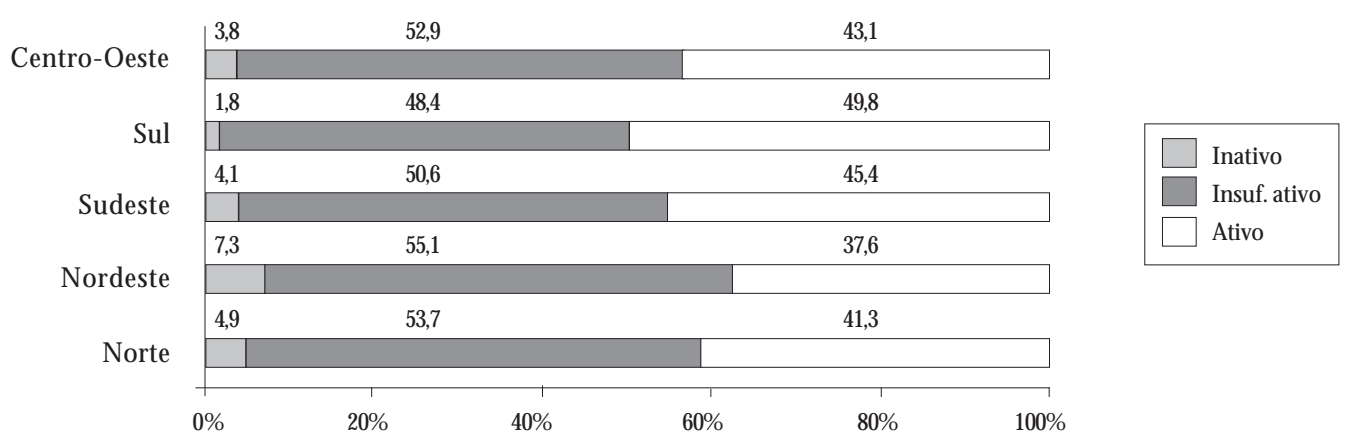

Gráfico 2. Proporção de jovens inativos, insuficientemente ativos e ativos conforme regiões do Brasil. Pesquisa Nacional de Saúde do Escolar, IBGE.

Tabela 1. Proporção de jovens inativos, insuficientemente ativos e ativos conforme sexo, escolaridade materna e tipo de escola. Pesquisa Nacional de Saúde do Escolar, IBGE.

\begin{tabular}{lccc}
\hline \multirow{2}{*}{$\begin{array}{c}\text { Variáveis } \\
\text { sociodemográficas }\end{array}$} & \multicolumn{3}{c}{ Atividade física de jovens (\%) } \\
\cline { 2 - 4 } & Inativo & Insuficientemente ativo & Ativo \\
\hline Sexo & & $p<0,001$ & 56,2 \\
$\quad$ M asculino & 2,7 & 41,1 & 31,3 \\
Feminino & 6,6 & 62,1 & 41,0 \\
Escolaridade materna & & $p<0,001$ & 43,9 \\
$\quad$ Fundamental incompleto & 5,0 & 54,0 & 43,1 \\
M édio incompleto & 4,5 & 51,6 & 50,4 \\
Superior incompleto & 4,6 & 52,3 & \\
Superior completo & 3,5 & 46,1 & 45,1 \\
Tipo de escola & & $p=0,14$ & 42,6 \\
$\quad$ Privada & 4,5 & 50,4 & 52,6 \\
Pública & 4,8 & & \\
\hline
\end{tabular}


A Tabela 3 mostra que a partici pação em aulas de educação física na semana anterior à entrevista foi ligeiramente superior nos meninos em comparação com as meninas, mas não variou conforme a escolaridade materna. Novamente, chama a atenção a homogeneidade no desfecho entre os grupos das exposições; em qualquer grupo analisado, apenas cerca de metade dos escolares relatou ter participado de duas ou mais aulas de educação física na semana anterior à entrevista. N a comparação por dependência administrativa escolar, apesar de os alunos de escolas públicas apresentarem percentual mais el evado de participação nas aulas na análise bruta ( Tabela 3), essa associação não foi mantida após ajuste para escolaridade materna (dados não mostrados).

\section{Discussão}

A mensuração de atividade física, especialmente em jovens, é desafiadora. Existem evidências de que os dados coletados por questionários concordam apenas parcialmente com aqueles oriundos de métodos mais diretos, especialmente os acelerômetros. Sabe-se, porém, que o monitoramento da prática de atividade física por meio de acelerometria seria praticamenteinviável em um país de renda média como o Brasil. Além disso,

Tabela 2. Proporção de jovens que assistem televisão menos de duas horas e duas horas ou mais por dia conforme sexo, escolaridade materna e tipo de escola. Pesquisa Nacional de Saúde do Escolar, IBGE.

\begin{tabular}{|c|c|c|}
\hline \multirow{2}{*}{$\begin{array}{c}\text { Variáveis } \\
\text { sociodemográficas }\end{array}$} & \multicolumn{2}{|c|}{ Tempo assistindo TV (\%) } \\
\hline & Duas horas ou mais por dia & Menos de duas horas por dia \\
\hline Sexo & \multicolumn{2}{|c|}{$p=0,89$} \\
\hline Masculino & 79,4 & 20,6 \\
\hline Feminino & 79,5 & 20,5 \\
\hline Escolaridade materna & \multicolumn{2}{|c|}{$p<0,001$} \\
\hline Fundamental incompleto & 78,9 & 21,1 \\
\hline M édio incompleto & 81,3 & 18,7 \\
\hline Superior incompleto & 81,6 & 18,4 \\
\hline Superior completo & 77,1 & 22,9 \\
\hline Tipo de escola & \multicolumn{2}{|c|}{$p=0,002$} \\
\hline Privada & 77,5 & 22,5 \\
\hline Pública & 80,0 & 20,0 \\
\hline
\end{tabular}

Tabela 3. Número de aulas semanais de educação física na última semana nas quais os jovens participaram conforme sexo, escolaridade materna e tipo de escola. Pesquisa Nacional de Saúde do Escolar, IBGE.

\begin{tabular}{|c|c|c|}
\hline \multirow{2}{*}{$\begin{array}{l}\text { Variáveis } \\
\text { sociodemográficas }\end{array}$} & \multicolumn{2}{|c|}{$\begin{array}{l}\text { Participação em aulas semanais de educação física } \\
\text { na semana anterior à entrevista }\end{array}$} \\
\hline & Duas aulas ou mais & Uma ou nenhuma aula \\
\hline Sexo & \multicolumn{2}{|c|}{$p<0,001$} \\
\hline Masculino & 50,8 & 49,2 \\
\hline Feminino & 47,8 & 52,2 \\
\hline Escolaridade materna & \multicolumn{2}{|c|}{$p=0,48$} \\
\hline Fundamental incompleto & 49,0 & 51,0 \\
\hline M édio incompleto & 49,5 & 50,5 \\
\hline Superior incompleto & 47,7 & 52,3 \\
\hline Superior completo & 50,6 & 49,4 \\
\hline Tipo de escola & \multicolumn{2}{|c|}{$p=0,06$} \\
\hline Privada & 43,9 & 56,1 \\
\hline Pública & 50,6 & 49,4 \\
\hline
\end{tabular}


existem evidências de que apesar de os escores contínuos dos questionários não concordarem com os escores contínuos oriundos de acelerometria, a categorização dos indivíduos em grupos de atividade física apresenta concordância moderada a elevada entre os instrumentos ${ }^{15}$, 0 que suporta a utilização de questionários em sistemas de monitoramento.

A elevada exposição à TV entreescolares brasileiros do $9^{\circ}$ ano é um achado impactante. Um estudo de coortena N ova Zelândia ${ }^{16}$ revelou que exposição excessiva à TV na infância ena adolescência se associa a tabagismo, baixa aptidão físi$\mathrm{ca}$, sobrepeso e colesterol el evado na vida adulta. Programas escolares que utilizassem maior tempo das crianças em atividades extraclasse poderiam minimizar a exposição a comportamentos sedentários como o hábito de assistir à TV.

A reduzida participação em aulas de educação física não é um fenômeno isolado em nosso país. Conforme os resultados do YRBSS para os Estados Unidos ${ }^{17}$, apenas $55 \%$ dos alunos do ensino médio estavam matriculados em aulas de educação física em 2003. Somente $28 \%$ destes frequentavam aulas de educação física diariamente. Um maior envolvimento dos alunos, focado principalmente em aulas que despertem seu interesse, parece ser um compromisso e desafio para a rotina de escolas e professores. Infelizmente, 0 instrumento de pesquisa utilizado na PeN SE, assim como a maior parte dos instrumentos disponíveis na área, não écapaz de compreender os motivos pelos quais vários adolescentes se afastam das aulas de educação física na escola; tais informações são extremamente relevantes e podem ser algo de investigações futuras.

Uma recente revisão sistemática da literatura latino-americana na área de interven ções em atividade física concluiu que a promoção de atividadefísica no ambienteescolar éaltamente recomendada e efetiva ${ }^{18}$. Exemplos como o Saúde na Boa, uma intervenção com estudantes do ensino noturno dos estados de Santa Catarina e Pernambuco, devem ser replicados em outros locais do país ${ }^{19,20}$. Pesquisadores, instituições acadêmicas, não governamentais e governamentais que visam promover a atividade física no Brasil devem urgentemente incluir a promoção da saúde na escola em suas agendas.

No entanto, a proposição de intervenções com jovens tendo como foco exclusivo o ambiente escolar deve ser encarada com cautela, especialmente se há interesse em resultados imediatos. Estudos de revisão apontam diversos aspectos como determinantes para a atividade física de jovens, não somente o espaço escolar. 0 estudo de Sallis et al. ${ }^{21}$ indica que 0 suporte social de pais, irmãos e pares é fundamental na adoção de hábitos mais ativos. A revisão de Seabra et al. ${ }^{22}$ confirma esses achados e salienta a questão socioeconômica, traduzi da na oportunidade de práticas em diferentes espaços. Portanto, intervenções focadas na escola podem ter sua efetividade limitada, se não considerarem diversos outros aspectos relacionados ao comportamento ativo.

0 ambiente físico escolar tem sido alvo de pesquisas recentes. Essa abordagem discute as políticas escolares com relação aos aspectos de oportunidade de equipamentos, espaços fiscos adequados, qualidade das aulas de educação física, tipo de construção e comportamento sedentário durante o período escolar. M esmo sendo um campo ainda carente de consistência em seus instrumentos, os resultados são muito interessantes no que diz respeito à formulação de intervenções específicas dentro do ambiente escolar. Pré-escolas e escolas de ensino fundamental e médio cujos ambientes físicos são mais adequados têm alunos mais ativos ${ }^{23}$. A parecem no escopo desse ambiente a existência de ginásios, quadras esportivas, áreas verdes, políticas voltadas à saúde e à atividade física, a atividades extracurriculares, aperfeiçoamento de professores, entre outras. No sistema de monitoramento americano, 0 ambienteescolar éavaliado a cada seis anos ${ }^{24}$. $\mathrm{Na}$ PeNSE foi inserido um bloco sobre ambiente escolar, possibilitando avaliar os espaços físicos destinados à prática de atividade física na escola e no entorno, que será objeto de análise futura.

O relatório da PeNSE ${ }^{11}$, ao analisar a atividade física de forma dicotômica e sem ajuste por qualquer indicador socioeconômico, mostrou que alunos de escolas privadas eram mais ativos do que aqueles de escolas públicas ( $45,1 \%$ versus $42,6 \%$ ). Embora no presente estudo tenhamos realizado a análise com esse desfecho em três categorias e não tenhamos encontrado diferenças, repetimos a associação mencionada na publicação controlando para escolaridadematerna. Essa análise nos indica, após ajuste para escolaridade materna, que qualquer possível efeito do tipo de escola desaparece.

Para promover a atividade física e a saúde no meio escolar, será preciso atribuir aos comportamentos saudáveis significados e interesse dos jovens. N ão teremos um modelo de interven ção queatenda às diferentes culturas e regiões do país, porém é indispensável replicar e divulgar as experiências bem sucedidas. A mudança na baixa atividade física e o comportamento sedentário 
hoje frequentes passam pela busca de tornar 0 ambiente escolar, a aula de educação física e outros meios de convívio dos jovens espaços atrativos e dotados de significados.

Os primeiros dados de atividade física da PeNSE são muito bem-vindos. Em termos de monitoramento, eles servirão como linha debase para comparações futuras. Com o objetivo de garantir isso, éfundamental queo instrumento e os métodos do inquérito sejam mantidos. Além de fornecer os dados de base para o monitoramento da atividade física de jovens brasileiros, os resultados da PeN SE representam o primeiro estudo de basenacional sobre atividade física em adolescentes. 0 percentual de jovens inativos e insuficientemente ativos é tão elevado, e surpreendentemente homogêneo entre as regiões do país, que intervenções para a promoção de atividade física em adolescentes brasileiros tornamse urgentes e devem ser priorizadas, principalmente porque é sabido que a atividade física se reduz drasticamente no transcorrer da adolescência atéo início da vida adulta, etal queda ainda deverá ser vivenciada por esses jovens.

\section{Colaboradores}

PC HAllal, AG Knuth, DKA Cruz, MI M endes e DC M alta participaram, igualmente, de todas as etapas de elaboração do artigo. 


\section{Referências}

1. Hallal PC, Victora CG, Azevedo MR, Wells JC Adolescent physical activity and health: a systematic review. Sports M ed 2006; 36(12):1019-1030.

2. Azevedo MR, Araujo CL, Cozzensa da Silva M, $\mathrm{H}$ allal PC. Tracking of physical activity from adolescence to adulthood: a population-based study. Rev Saude Publica 2007; 41(1):69-75.

3. Bastos JP, Araujo CL, Hallal PC. Prevalence of insufficient physical activity and associated factors in Brazilian adolescents. Journal of Physical Activity \& Health 2008; 5(6):777-794.

4. Silva RC, Malina RM. Level of physical activity in adolescents from Niteroi, Rio de Janeiro, Brazil. Cad Saude Publica 2000; 16(4):1091-1097.

5. Hallal PC, Bertoldi AD, Gonçalves H, Victora CG. Prevalence of sedentary lifestyle and associated factors in adolescents 10 to 12 years of age. Cad Saude Publica 2006; 22(6):1277-1287.

6. Knuth AG, Hallal PC. Temporal trends in physical activity: a systematic review. Journal of Physical Activity \& Health 2009; 6(5):548-559.

7. Eaton DK, Kann L, Kinchen S, Shanklin S, Ross J, Hawkins J, Harris WA, Lowry R, M CM anus T, Chyen D, Lim C, Brener ND, Wechsler H. Centers for Disease Control and Prevention (CDC). Youth Risk Behavior Surveillance - United States, 2007. M M W R Surveill Summ 2008; 57(4):1-131.

8. Everett SA, Kann L, M cReynolds L. The Youth Risk Behavior Surveillance System: policy and program applications. The Journal of School Health 1997; 67(8):333-335

9. Florindo AA, Hallal PC, M oura EC, M alta DC. Practice of physical activities and associated factors in adults, Brazil, 2006. Rev Saude Publica 2009; 43(Suppl 2):65-73.

10. Tassitano RM, Bezerra J, Tenorio M C, Colares V, Barros MV, Hallal PC. Atividade física em adolescentes brasileiros: uma revisão sistemática. Rev Bras Cineantropom Desempenho H um 2007; 9(1):55-60.

11. Brasil, M inistério da Saúde, Instituto Brasileiro de Geografia e Estatística. Pesquisa Nacional de Saúde do Escolar. Rio de Janeiro: Ministério da Saúde, Instituto Brasileiro de Geografia e Estatística; 2009.

12. Tassitano RM, Barros MV, Tenorio M C, Bezerra J, $\mathrm{H}$ allal PC. Prevalence of overweight and obesity and associated factors among public high school students in Pernambuco State, Brazil. Cad Saude Publica 2009; 25(12):2639-2652.

13. Hallal AL, Gotlieb SL, Almeida LM, Casado L. Prevalence and risk factors associated with smoking among school children, Southern Brazil. Rev Saude Publica 2009; 43(5):779-788.

14. Biddle S, Cavill N, Sallis J. Young and active? Young people and health-enhancing physical activity - evidence and implications. London: Health Education Authority; 1998.
15. Hallal PC, Simões EJ, Reichert FF, Azevedo MR, Ramos LR, Pratt M, Brownson RC. Validity and reliability of the Telephone-Administered International Physical Activity Questionnaire in Brazil. Journal of Physical Activity \& Health 2010; 7:402-409.

16. Hancox RJ, Milne BJ, Poulton R. Association between child and adolescent television viewing and adult health: a longitudinal birth cohort study. Lancet 2004; 364(9430):257-262.

17. Participation in high school physical education United States, 1991-2003. M M WR 2004; 53(36):844847.

18. Hoehner CM, Soares J, Parra Perez D, Ribeiro IC, Joshu CE, Pratt M, Pratt M, Legetic BD, Malta DC, M atsudo VR, Ramos LR, Simões EJ, Brownson RC. Physical activity interventions in Latin America: a systematic review. Am J Prev M ed 2008; 34(3):224233.

19. Barros MV, Nahas MV, Hallal PC, de Farias Junior JC, Florindo AA, Honda de Barros SS. Effectiveness of a school-based intervention on physical activity for high school students in Brazil: the Saude na Boa project. Journal of Physical Activity \& Health 2009; 6(2):163-169.

20. Nahas MV, Barros MV, Assis M A, Hallal PC, Florindo AA, Konrad L. Methods and participant characteristics of a randomized intervention to promote physical activity and healthy eating among Brazilian high school students: the Saude na Boa project. Journal of Physical Activity \& Health 2009; 6(2):153162.

21. Sallis JF, Prochaska JJ, Taylor WC. A review of correlates of physical activity of children and adolescents. Medicine and Science in Sports and Exercise 2000; 32(5):963-975.

22. Seabra AF, Mendonca DM, Thomis MA, Anjos LA, $M$ aia JA. Biological and socio-cultural determinants of physical activity in adolescents. Cad Saude Publica 2008; 24(4):721-736.

23. Dowda M, Brown WH, Mclver KL, Pfeiffer KA, O'N eill JR, Addy CL, Pate RR. Policies and characteristics of the preschool environment and physical activity of young children. Pediatrics 2009; 123(2):261266.

24. Jones SE, Fisher CJ, Greene BZ, Hertz M F, Pritzl J. Healthy and safe school environment, part I: results from the School Health Policies and Programs Study 2006. Journal of School Health 2007; 77(8):522-543.

Artigo apresentado em 08/06/2010

Aprovado em 21/06/2010

Versão final apresentada em 02/08/2010 